\title{
A Framework for Bandwidth Management in ATM Networks-Aggregate Equivalent Bandwidth Estimation Approach
}

\author{
Zbigniew Dziong, Marek Juda, and Lorne G. Mason
}

\begin{abstract}
A unified framework for traffic control and bandwidth management in ATM networks is proposed. It bridges algorithms for real-time and data services. The central concept of this framework is adaptive connection admission. It employs an estimation of the aggregate equivalent bandwidth required by connections carried in each output port of the ATM switches. The estimation process takes into account both the traffic source declarations and the connection superposition process measurements in the switch output ports. This is done in an optimization framework based on a linear Kalman filter. To provide a required quality of service guarantee, bandwidth is reserved for possible estimation error. The algorithm is robust and copes very well with unpredicted changes in source parameters, thereby resulting in high bandwidth utilization while providing the required quality of service. The proposed approach can also take into account the influence of the source policing mechanism. The tradeoff between strict and relaxed source policing is discussed.
\end{abstract}

\section{INTRODUCTION}

$\mathbf{T}$ RAFFIC control and bandwidth management in ATM networks has attracted a lot of attention due to the potential for improved utilization through statistical multiplexing of traffic sources. In spite of considerable research effort, no universal solution to this problem has been found. The proposed approaches usually are suited to a particular type of traffic and in general can be divided into two categories. In the first one, the focus is on restricting the traffic entering the network based solely on the source type and declarations, regardless of the current level of traffic generated by the sources. A typical example is the equivalent bandwidth allocation based on source declarations and policing mechanisms (see, e.g., [1]-[5]). The second category covers algorithms which include some kind of dependence on the current state of the network. Congestion notification (see, e.g., [6]) and window mechanisms (see, e.g., [7], [8]) are examples of the second type. The limitations of the first category are source restrictions and design for the worst-case (potential for low

Manuscript received August 14, 1995; revised December 3, 1995, and April 11, 1996; approved by IEEE/ACM TRANSACTIONS ON NETwORKING Editor J. Roberts. This work was supported by the Canadian Institute for Telecommunications Research under the NCE program of the Government of Canada. This paper was presented in part at the IEEE INFOCOM'95, Conference on Computer Communications, 1995, and in part at the ITC-14, International Teletraffic Congress, 1994.

Z. Dziong and L. G. Mason are with INRS-Telecommunications, Verdun, Quebec, H3E 1H6 Canada (email: dziong@inrs-telecom.uquebec.ca; lorne@inrs-telecom.uquebec.ca).

M. Juda was with INRS-Telecommunications, Verdun, P.Q., H3E 1H6 Canada. He is now with Andersen Consulting, Warsaw, Poland.

Publisher Item Identifier S 1063-6692(97)01640-3. utilization). The second category is more flexible (potential for high utilization) but does not provide guarantees for throughput nor for quality-of-service (QoS).

These two approaches constitute almost two disjoint worlds. They are focused on different traffic sources (real-time versus controllable data) and the key elements of the algorithms have no common protocol or data base structure. In the first part of the paper (Section II), we propose a framework for traffic control and bandwidth management which bridges these two worlds. We start from a simple observation that since the congestion occurs at the switch output ports, any efficient and flexible traffic control mechanism should have access to some information about the current cell process in the switch output ports. Note that this is the case in most of the congestion control mechanisms for controllable data traffic where the switch output buffer states are measured directly (congestion notification mechanisms) or indirectly (window mechanisms). We argue that direct measurement of the cell process in the switch output port can constitute a common base for traffic control and bandwidth management of both real-time and controllable data services.

For the controllable data services we adopt the rate-based approach recommended by the ATM Forum (see, e.g., [6]). This adaptive scheme explicitly regulates the source rates by means of the switch output buffer state measurements and resource management cells. For the real-time services we propose an approach based on estimation of the aggregate equivalent bandwidth required by all connections served by each of the switch output ports. In order to use all available relevant information, the estimation procedure employs the source parameter declarations and direct measurement of the connection superposition cell process parameters in the switch output ports. In this scheme, the connection admission procedure can verify the available bandwidth on all considered links by means of a resource management cell passing through the transit nodes. One of the key advantages of the proposed framework is that the traffic control and bandwidth management algorithms for real-time and controllable data services are based on the same data base structure and signaling protocol. Besides reduction in algorithms complexity and cost, this feature can also increase bandwidth utilization by enabling coordination of both algorithms. Another important feature of the proposed scheme is that it is not restricted to any particular link or source model. In other words, any reasonable model for analytical evaluation of the connection equivalent bandwidth can be used in this framework. 
In the literature, one encounters other connection admission algorithms based on measurements [9]-[14]. They differ in many aspects from our framework. For example, the mechanism described in [9] measures individual source process parameters which are then used to adapt source policing mechanism parameters and the equivalent bandwidth required by the connection. On the other hand, the algorithm proposed in [10] uses measurements of the superposition cell process to estimate the cell arrival distribution but does not operate in the equivalent bandwidth domain. In [12] and [13], Bernoulli link models and Bayesian decision theory are used for connection admission based on the measured link load. In [14], it is observed that measurements of the cell process in the low frequency band can constitute a basis for adaptive connection admission control.

In the second part of the paper, we design and analyze a particular model for connection admission based on aggregate equivalent bandwidth estimation in the switch output ports. The estimation process is decomposed into two parts (Section III). First the algorithm estimates certain parameters of the link connection superposition process. Then, based on the estimated and declared parameters, the aggregated equivalent bandwidth is estimated. The estimation of the chosen parameters (mean and variance of the instant cell rate process) employs a two-state linear Kalman filter (other applications of a Kalman filter for network management can be found in [15] and [16]). The important feature of the Kalman filter model is that it provides information about the estimation error in terms of the declaration and measurement errors. This information is used to evaluate bandwidth reserved for estimation errors in order to provide statistical guarantees for the QoS. The reserved bandwidth enables the source parameter declarations to be more relaxed and the source policing less stringent compared to equivalent bandwidth allocation based solely on the source parameter declarations. Concerning the ATM Forum and ITU recommendations for source parameter declaration, the proposed approach can be seen as complementary. In particular, the predicted connection traffic parameters, if not declared, can be estimated by the network operator based on the declared policing parameters, source type and long term statistics concerning this type of source.

The accuracy of the estimation process is analyzed in three stages (Section IV). First, we concentrate on the evaluation of the measurement error. Then we analyze the errors of estimates provided by the Kalman filter. Finally, we verify the accuracy of the model used for estimation of the aggregate bandwidth and the bandwidth reserved for the estimation error. The results show that, for the Gaussian declaration model, the estimation process is very accurate. The accuracy of the whole connection admission algorithm is verified under nonstationary conditions and large, non-Gaussian, declaration errors (Section V). The results demonstrate that the algorithm copes very well with unpredicted changes in source parameters by providing high bandwidth utilization and the required QoS. In Section VI, we extend the estimation model to take into account the influence of source policing mechanisms. The numerical study of this option illustrates the trade-off between strict and relaxed source policing.

\section{FRAMEWORK FOR UNIFIED TRAFFIC CONTROL AND BANDWIDTH MANAGEMENT}

The issue of traffic control and bandwidth management in ATM based networks is complex due to a mixture of different connection traffic types, QoS requirements and time scales. In this section, we describe a framework which provides a coherent and effective structure for traffic control and bandwidth management of all services.

We start from a classification of the service categories. According to the ATM-Forum recommendations (see, e.g., [6]) one can recognize five service categories at the ATM layer: constant bit rate (CBR), real-time variable bit rate (VBR-RT), non-real-time variable bit rate (VBR-NRT), available bit rate $(\mathrm{ABR})$, and unspecified bit rate (UBR). From the bandwidth management and traffic control viewpoint, these categories can be aggregated into two macro-categories: controllable traffic parameters $(\mathrm{CTP}=\mathrm{ABR})$ and noncontrollable traffic parameters $(\mathrm{NCTP}=\mathrm{CBR}+\mathrm{VBR}-\mathrm{RT}+\mathrm{VBR}-\mathrm{NRT})$. Note that the UBR category is not included in the macro-categories. This follows from the fact that UBR services have smallest priority on the cell layer and do not require any additional traffic admission control or resource allocation.

In the following, we present a framework which, to a large extent, can unify data base structure, signaling protocols and algorithms for traffic control and bandwidth management of NCTP and CTP services. There are four principles from which the framework is derived. The algorithms should operate explicitly in terms of the bandwidth so the cooperation between bandwidth management algorithms on the connection and higher layers is straightforward. All available information should be used in an optimal way (in particular, it means that the algorithms should use explicit measurements of the relevant network state and source traffic parameters declarations). The algorithms should not be restrictive in the sense that even an unpredicted traffic increase should not be rejected if there is available bandwidth. Finally, the algorithms for different service categories (CTP and NCTP) should be based on the same data base structure and similar signaling protocols in order to reduce complexity and cost of traffic control and bandwidth management.

We adopt the ATM Forum recommendation for traffic control of the CTP (ABR) service class (see, e.g., [6]). The basic idea of this rate-based recommendation is illustrated in Fig. 1(a). During a connection, at least every $100 \mathrm{~ms}$, the source sends to the destination a resource management (RM) cell with information about the current cell rate, CCR. At each node, the bandwidth management algorithm evaluates the desired rates (DR) for each virtual channel (VC) based on the declared CCR's (received from RM cells) and measurements of the cell process parameters, $Z$, in the switch output ports used by VC's. The desired rates are inserted into the passing RM cells which are returned to the source by the destination node. If the RM cell is lost, the source automatically reduces the rate. Note that one can establish a simple relation between the connection rate and the equivalent bandwidth required by the connection. Thus, the rate-based algorithm is in accordance with the first three adopted principles since it can operate in 


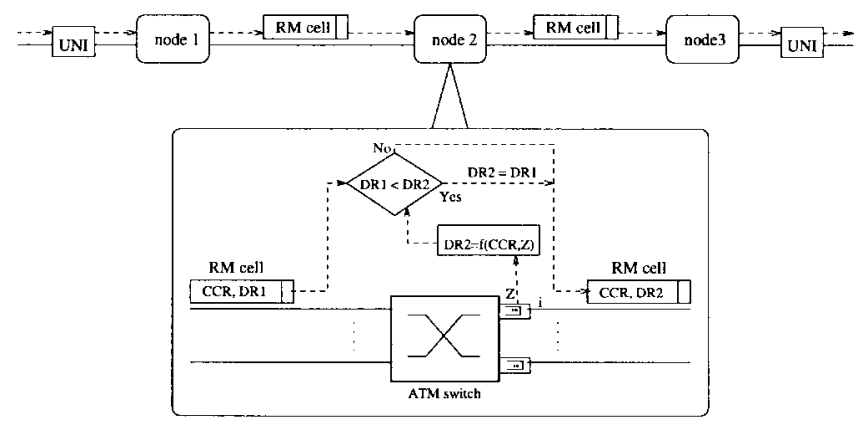

(a)

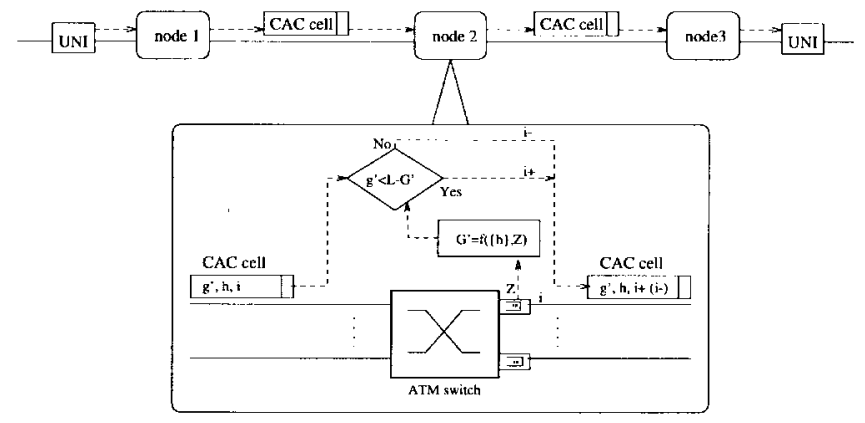

(b)

Fig. 1. TR\&BM mechanism for (a) CTP services and (b) NCTP services.

the bandwidth domain, uses source declarations and explicit measurements of the relevant network state, and allows a rate increase whenever there is available bandwidth.

In the literature, one finds several propositions for logical bandwidth allocation to virtual channels for the NCTP category of services (e.g., [19], [4], [5], [20], [21]). The most attractive are the ones employing equivalent bandwidth allocation since in this case the gain from statistical multiplexing can be realized. The problem with most algorithms employing the equivalent bandwidth notion is that they are based only on the source declared parameters and the policing mechanism. Since it can be difficult for some sources to predict their traffic parameters in advance and it might be hard to enforce declarations of some statistical parameters, the scheme is restrictive and prone to under-utilization of the bandwidth (design for the worst-case). In order to avoid these drawbacks and fulfill all the objectives of the adopted principles, we propose an approach which is illustrated in Fig. 1(b). In response to the new connection request, the connection admission algorithm at the user-network interface (UNI) sends a resource management cell (henceforth referred to as CAC cell) to the destination UNI along the path recommended by the routing algorithm. The cell contains information about the declared traffic parameters, $h$, and required equivalent bandwidth, $g^{\prime}$. In each node, the traffic control and bandwidth management algorithm estimates the aggregate equivalent bandwidth, $G^{\prime}$, which should be reserved for all connections carried on each of the outgoing links. This is carried out using the measurements of the superposition cell process parameters, $Z$, in the switch output ports and the source declared traffic parameters, $\{h\}$, of the connections already accepted. When a CAC cell arrives at the node, the algorithm verifies whether the requested bandwidth, $g^{\prime}$, is smaller than (or equal to) the residual bandwidth, $C=L-G^{\prime}$. This information is inserted into the passing CAC cell and the bandwidth is reserved if $g^{\prime} \leq C$. At the destination UNI, the cell is returned to the origin UNI in order to reserve the bandwidth on the return path. Obviously the proposed algorithm can also be implemented by using the standard BISUP protocol for connection set-up [22]. Nevertheless, using resource management cells and the protocol derived for flow control of CTP services (on the pre-established virtual path recommended for the connection) has several advantages, the most important being reduction of connection set-up time and integration with algorithms for CTP services.

The central idea of the approach for NCTP services is a simple observation that the CAC algorithm does not need to know precise values of the equivalent bandwidth required by each individual connection. On the contrary, it is the cell superposition process which defines the quality of service and that is why the aggregate equivalent bandwidth is employed as the main control variable. Another advantage of this approach is that the scheme can be much less restrictive compared to the schemes based on policing mechanisms. This is caused by two factors, namely, the statistical multiplexing of the bandwidth allocation errors and fast adaptability. The first factor concerns the problem of the bandwidth reservation for the worst-case when a policing mechanism enforces bandwidth allocation to the source. This reservation has to take into account possible errors in declared traffic parameters. Although one can claim that the same problem exists with the aggregated bandwidth, it can be easily shown that the standard deviation of the aggregated process parameter error is in general significantly smaller than the sum of the standard deviation of individual connection traffic parameter errors. The second factor relates to the fact that measurement of the cell superposition process is much more reliable than the sum of each connection process measurements (this can again be explained by statistical multiplexing of the measurement errors). This feature ensures that even if there is a large declaration error it will be quickly reduced by adjusting the bandwidth allocation. Thanks to both effects, the source policing can be significantly relaxed and the interpretation of its function is changed from bandwidth enforcement to controlling the magnitude of the aggregate bandwidth estimation error.

Comparison of the traffic control and bandwidth management structures for CTP and NCTP services (Fig. 1) shows that both approaches employ the same data base structure and signaling protocol. In addition to significant reduction of the bandwidth management algorithm complexity and cost, this feature can also increase bandwidth and buffer utilization by close cooperation of both algorithms. For example, the explicit rate allocation algorithms for CTP connections would be more efficient and stable if parameters of the aggregated cell process of NCTP services could be predicted in advance. Since this prediction is an inherent feature of the proposed connection admission for NCTP services, both algorithms may use a common prediction algorithm where some parts would be parameterized according to the needs of each of the two applications (e.g., to accommodate differences in the considered time horizon). In the remainder of this paper, 
we consider implementation of the proposed framework for connection admission for NCTP services only. First, we focus on an aggregate equivalent bandwidth estimation model and its error analysis. Then, the connection admission control mechanism is studied.

\section{Model FOR AGGREgATE EQUIVALENT BANDWIDTH ESTIMATION}

In the proposed framework, an estimate of the aggregate equivalent bandwidth, $\hat{G}$, is a function of the declared parameters of accepted connections, $H=\left\{h_{j}\right\}$, and measured parameters, $Z$, of the connection superposition process at the switch output port. It is obvious that the estimate $\hat{G}$ may be different from the real value, $G$, due to the declaration and estimation errors. If we assume that the bandwidth reserved for accepted connections, $G^{\prime}$, equals $\hat{G}$, the QoS constraints can be violated with high probability. In order to keep this probability at an acceptable level, a bandwidth, $R$, reserved for the estimation error is introduced so the connection acceptance rule is based on the bandwidth reserved for aggregate traffic defined as

$$
G^{\prime}=\hat{G}+R .
$$

Thus the objective of the estimation process is to find $\hat{G}$ and $R$ such that

$$
P\{G>\hat{G}+R\} \leq \epsilon_{1}
$$

where $\epsilon_{1}$ is the estimation error constraint.

Note that from the connection admission point of view, we are interested in discrete points of time. Thus, the system is modeled in the discrete time domain where $t_{k}, k=1,2, \cdots$ denotes the instant of the system state change, $X_{k-1} \rightarrow X_{k}$, caused either by a new connection, or by a connection release. In general, the system state can be defined by some parameters being a function of the cell rate superposition process, $S(t)$. Based on estimation theory, it can be shown that, by applying a recursive discrete filter, the state estimate of our system, $\hat{X}_{k}$, and the covariance matrix of its error, $P_{k}$, can be evaluated as a function of the following parameters: $h_{k}, Z_{k}, \hat{X}_{k-1}$ (where $h_{k}$ denotes the declared parameters of the connection added or released in the transition $X_{k-1} \rightarrow X_{k}$ ). In addition, the parameters, $q_{k}, Y_{k}$, defining the declaration and measurement error distributions (assumed to be Gaussian) are required. The issue of $\hat{G}_{k}$ and $R_{k}$ estimation fits very well into the framework of recursive discrete filters which suggests that the natural choice for the state description would be the aggregate equivalent bandwidth, $G_{k}$, so the required estimates, $\hat{G}_{k}, R_{k}$, would be achieved directly. While this approach is possible, in the following we propose another state description which simplifies the estimation process and ensures that it is not limited to a particular model for equivalent bandwidth evaluation.

There are two difficulties with direct estimation of $\hat{G}_{k}$. The relationship between the equivalent bandwidth and the parameters which can be directly measured, $\hat{G}_{k}=f_{\hat{G}}\left(Z_{k}\right)$, is in general nonlinear. This suggests that a nonlinear filter should be applied which is typically more complex than a linear

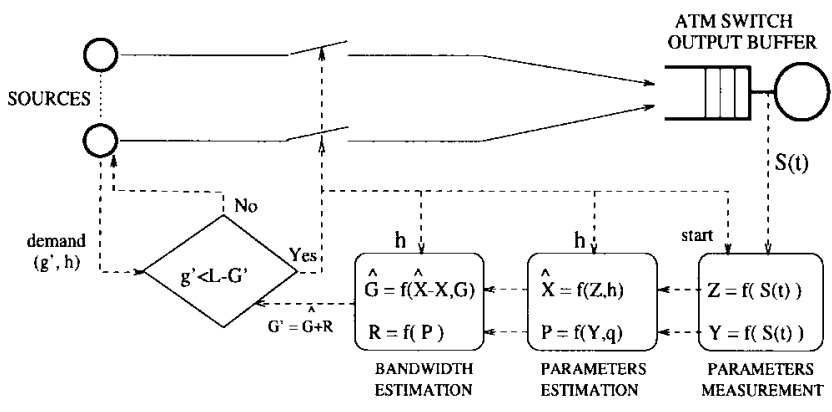

Fig. 2. Structure of the control system.

filter. Although in the literature one can find several models for evaluation of the equivalent bandwidth, these algorithms are relatively complex and do not provide a universal closed form solution. These characteristics significantly increase the complexity of the problem, since the function $f_{\hat{G}}$ must be inverted in the estimation algorithm.

To avoid these problems, we chose a vector of the superposition cell rate process parameters as the state description which can be directly measured at the switch output port. Thus a linear filter can be applied and the inverse function problem becomes trivial. Having the estimate of the system state, $\hat{X}_{k}$, the estimate of aggregate equivalent bandwidth can then be evaluated from a differential approach

$$
\hat{G}_{k}=f_{\Delta \hat{G}}\left(\hat{X}_{k}-X_{k}^{d}, G_{k}^{d}\right)
$$

where $X^{d}, G^{d}$ denote the values evaluated from the declared parameters. The differential approach provides that the proposed CAC algorithm can work with any reasonable model for evaluation of the connection equivalent bandwidth since this model is not directly used in the estimation procedure. The structure of the control system based on the differential approach is illustrated in Fig. 2.

In the remainder of the paper, we describe and investigate a particular version of the proposed scheme where mean and variance of the instant cell rate of the connection superposition process are chosen as the system state description and measured parameters. While at first glance it might be viewed as a bold simplification due to the lack of any temporal characterization, observe that the equivalent bandwidth can be evaluated from the full set of declared parameters, $h_{k}$, which take into account all important source features while the estimated parameters serve only to correct this bandwidth allocation. The implied assumption is that, in general, the sources do not change their basic features associated with the type of the connection (otherwise a more sophisticated set of estimated and measured parameters would have to be chosen). The choice of the instant cell rate mean and variance is also supported by the burst scale performance analysis (see, e.g., [19]). This analysis indicates that in a robust CAC algorithm for real-time applications the arrival link cell rate should only exceed link capacity with a very small probability while the buffer dimensioning should take care of the cell scale congestion (small time scale variability factor). In this case the temporal characterization of the cell rate process is not critical. Obviously, in the (unlikely) case of very large buffers the 
chosen parameters should be complemented by a parameter(s) characterizing the cell rate autocorrelation function.

Concerning the source declaration parameters $h_{k}$ we assume that they include the predicted connection average cell rate, $m_{k}^{d}$, cell rate variance, $v_{k}^{d}$, and variances of these prediction errors, $q_{k}=\left[v_{e, k}^{m}, v_{e, k}^{v}\right]^{T}$, respectively. These values can be declared directly by the source or can be estimated based on the source type, declared policing parameters (peak rate, sustained rate, and burst tolerance), and long term statistics concerning this source type behavior. It should be underlined that the study presented in this paper focuses on the proposed estimation procedure and adaptive connection admission control. Since these algorithms are independent from the model for equivalent bandwidth evaluation based on the connection declared parameters, we do not discuss such models and throughout the paper we assume that the function for equivalent bandwidth evaluation is given, $g_{i}^{d}=f_{g}\left(h_{i}\right)$. The reader interested in models for equivalent bandwidth evaluation is referred to a substantial literature on this subject (e.g., [1]-[5], [19]-[21]).

\section{A. Estimation of the Cell Rate Mean and Variance}

The objective of the estimation process is to provide the best estimate of the mean, $M_{k}$, and variance, $V_{k}$, of the instant cell rate of the connection superposition process. In this paper, we treat these two variables as independent although the proposed approach can also take into account the correlated case. The state of our system is defined as $X_{k}=\left[M_{k}, V_{k}\right]^{T}$. The dynamics of the system model is illustrated in Fig. 3 and is described by

$$
X_{k}=X_{k-1}+x_{k}+e_{k}
$$

where $e_{k}$ denotes the model error and $x_{k}=\left[\alpha_{m} m_{k}, \alpha_{v} v_{k}\right]^{T}$ denotes either the declared mean and variance of the accepted connection $\left(\alpha_{m}=\alpha_{v}=1\right)$ or the normalized declared mean and variance of the released connection $\left(\alpha_{m}=\right.$ $-\hat{M}_{k} / M_{k}^{d}, \alpha_{v}=-\hat{V}_{k} / V_{k}^{d}$, where index $d$ denotes parameters evaluated from declarations). For the time being we assume that the model error, $e_{k}=\left[\delta_{k}^{m}, \delta_{k}^{v}\right]^{T}$, is a Gaussian random variable with zero mean and covariance matrix, $Q_{k}$. The interpretation of the model error distribution parameters and their evaluation based on the declaration error parameters, $q_{k}$, are discussed in Section IV-B.

The system model in Fig. 3 is complemented by the measurement model which provides the measurement of the cell rate mean and variance in state $k, Z_{k}=\left[\bar{M}_{k}, \bar{V}_{k}\right]^{T}$. The delay corresponds to the fact that the result of measurement of the parameters is required at the time of the next state change. The measurement model is defined as follows

$$
Z_{k}=X_{k}+u_{k}
$$

where $u_{k}=\left[\bar{\delta}_{k}^{m}, \bar{\delta}_{k}^{v}\right]^{T}$ is the measurement error. The measurement error is assumed to be a Gaussian random variable with zero mean and known covariance matrix, $Y_{k}$. The evaluation and interpretation of the measurement error parameters are discussed in Section IV-A.

The system and measurement model fit very well into the framework of linear recursive filters. In the following



Fig. 3. System model and Kalman filter.

we demonstrate application of the linear discrete Kalman filter (see, e.g., [23]) to the considered system. In general, the Kalman filter provides an optimal least-square estimate of the system state for a linear system and the model and measurement errors are Gaussian random variables. The block diagram of the applied filter is shown in Fig. 3. The system state estimate update is given by

$$
\hat{X}_{k}=\hat{X}_{k}^{e}+K_{k}\left[Z_{k}-\hat{X}_{k}^{e}\right]
$$

where $\hat{X}_{k}^{e}=\hat{X}_{k-1}+x_{k}$ denotes the state estimate extrapolation and $K_{k}$ is the Kalman filter gain. From the form of (6), it is clear that the gain is a weight which decides how much confidence should be given to the measurement versus the declaration.

To simplify notation let us introduce the transition matrix, $F_{k}$, with the following elements:

$$
a_{11}=\frac{M_{k-1}+\alpha_{m} m_{k}}{M_{k-1}}, \quad a_{22}=\frac{V_{k-1}+\alpha_{v} v_{k}}{V_{k-1}}
$$

and $a_{12}=a_{21}=0$. Thus, the system model can be described by

$$
X_{k}=F_{k} X_{k-1}+e_{k} .
$$

The Kalman gain is defined by

$$
K_{k}=P_{k}^{e}\left[P_{k}^{e}+Y_{k}\right]^{-1}
$$

where $P_{k}^{e}$ denotes the estimate error covariance matrix extrapolation given by

$$
P_{k}^{e}=F_{k-1} P_{k-1} F_{k-1}^{T}+Q_{k}
$$

where $P_{k-1}$ is updated estimate error covariance matrix evaluated from

$$
P_{k-1}=\left[I-K_{k-1}\right] P_{k-1}^{e} .
$$

Note that under the Gaussian assumption of the model and measurement errors, the estimation error distributions are also Gaussian with zero mean and variance defined by diagonal elements of the error covariance matrix, $\hat{v}_{e, k}^{m}, \hat{v}_{e, k}^{v}$, for cell rate mean and variance, respectively. These variances can be used to evaluate the bandwidth $R_{k}$ reserved for the error in 
evaluation of $\hat{G}_{k}$. The details of the procedure are given in the next section. Concerning the initial values we assume that the system is empty at the time $k=0$ so $X_{0}=0$ and $P_{0}=0$.

\section{B. Estimation of Aggregate Equivalent Bandwidth}

As indicated, we assume that the function for equivalent bandwidth evaluation from the declared parameters is given $g_{i}^{d}=f_{g}\left(h_{i}\right), G_{k}^{d}=\Sigma_{i} g_{i}^{d}$. In the following, we focus on estimation of the aggregate equivalent bandwidth from the differential approach defined by (3). In this case one needs to evaluate the sensitivity of $G$ with respect to the cell rate mean and variance around the declaration point $G^{d}$ (to simplify presentation the time index is omitted in this section). Note that the sensitivity function should not be complex since it is used in on-line evaluations. To fulfill this requirement, we derive this function from a simple expression suggested for approximate equivalent bandwidth evaluation (see, e.g., [19], [24], [25])

$$
g^{d}=\gamma \cdot m^{d}+\theta \cdot v^{d} .
$$

Based on (11), for a particular state of accepted connections, we have

$$
G^{d}=\gamma \cdot M^{d}+\theta \cdot V^{d}
$$

Since the values, $G^{d}, M^{d}$, and $V^{d}$ are known for each link state, one can evaluate parameters $\gamma, \theta$ from two recent link states. Alternatively, one can assume that the coefficient $\gamma$ is independent from the current state and can be evaluated offline using the link speed, QoS constraint, and average traffic mixture. Then the coefficient $\theta$, for a given state, is given by

$$
\theta=\frac{G^{d}-\gamma \cdot M^{d}}{V^{d}}
$$

Finally, the estimated equivalent bandwidth of the connection superposition process is evaluated from

$$
\hat{G}=\gamma \cdot \hat{M}+\theta \cdot \hat{V}
$$

It should be emphasized that, contrary to its appearance, (14) serves only for evaluation of the correction to the declared equivalent bandwidth $G^{d}$ implied by deviation of the estimated parameters, $\hat{M}, \hat{V}$, from the declarations, $M^{d}, V^{d},\left(G^{d}, M^{d}, V^{d}\right.$ and the function for equivalent bandwidth allocation are hidden in $\gamma$ and $\theta$ ).

Concerning the bandwidth reserved for the estimation error, under the Gaussian assumptions of the estimated mean and variance errors, the estimated bandwidth error, $\delta_{G}=\hat{G}-G$, is also Gaussian. Thus, under the assumption of mean and variance independence we have

$$
R=U\left(\epsilon_{1}\right) \sqrt{\gamma^{2} \hat{v}_{e}^{m}+\theta^{2} \hat{v}_{e}^{v}}
$$

where $\hat{v}_{e}^{m}, \hat{v}_{e}^{v}$ denote diagonal elements of the error covariance matrix, $P$, and $U\left(\epsilon_{1}\right)$ denotes a coefficient derived from the normalized Gaussian distribution which ensures that

$$
P\{G>\hat{G}+R\} \leq \epsilon_{1} .
$$

\section{ERROR ANALYSIS}

\section{A. Measurement Error}

An optimal cell process measurement is complex and should involve analysis of the autocorrelation function (see, e.g., [26]). This issue is large enough to be treated in a separate publication. In this paper, we adopt an approach which is simple to implement but still enables the analysis of important features of the proposed traffic admission control model.

In general, the measurement process can be divided into two stages. The first one provides information concerning the instant cell rate. In the paper we assume that this estimate is given. In the second stage, mean $M_{k}$, variance $V_{k}$ of the instant rate and the measurement errors are estimated. In the following, we analyze a standard approach based on instant cell rate samples, $\left\{d_{i}\right\}$, taken in regular intervals in the period $\left[t_{k}, t_{k+1}\right)$ and assumed to be independent. (A more general approach based on the autocorrelation function of the instant cell rate process would require estimation of the autocorrelation function, possibly using declarations and measurements.)

The standard estimates of the measured cell rate mean and variance are given by

$$
\begin{aligned}
\bar{M}_{k} & =\frac{\sum_{i} d_{i}}{N_{k}} \\
\bar{V}_{k} & =\frac{\sum_{i}\left(d_{i}-\bar{M}_{k}\right)^{2}}{N_{k}-1}
\end{aligned}
$$

where $N_{k}$ denotes the number of the samples. Let us define the measurement errors for mean and variance of the instant rate as

$$
\begin{aligned}
\bar{\delta}_{k}^{m} & =\bar{M}_{k}-M_{k} \\
\bar{\delta}_{k}^{v} & =\bar{V}_{k}-V_{k}
\end{aligned}
$$

respectively. The theoretical values of these error variances are given by

$$
\begin{aligned}
& v_{e, k}^{m}=\frac{V_{k}}{N_{k}} \\
& v_{e, k}^{v}=\frac{S_{k}-\left(V_{k}\right)^{2}}{N_{k}}
\end{aligned}
$$

where $V_{k}, S_{k}$ denote the variance and forth central moment of an instant rate, respectively. Since in our framework only measured values are available we apply the following estimates:

$$
\begin{aligned}
& \bar{v}_{e, k}^{m}=\frac{\bar{V}_{k}}{N_{k}} \\
& \bar{v}_{e, k}^{v}=\frac{\bar{S}_{k}-\left(\bar{V}_{k}\right)^{2}}{N_{k}}
\end{aligned}
$$

where

$$
\bar{S}_{k}=\frac{\sum_{i}\left(d_{i}-\bar{M}_{k}\right)^{4}}{N_{k}-1} .
$$

In the following, we analyze the accuracy of the approach by means of the connection admission process simulation at one switch output port (the details of the simulation model 
are given in Section V). The selected example is defined by the following parameters: link capacity $L=25$, connection requests intensity $\lambda=200^{-1}$, mean connection holding time $\mu^{-1}=5 \cdot 10^{4}$; source parameters-peak rate $P R=1$, fixed burst length $B=50$, exponentially distributed silence length with average $S=70$; simulation run-time- $4 \cdot 10^{6}$. The measurement quality is assessed for the case when the sources conform to the declared parameters. In this example, it is assumed that the sampling period, equal to the average on-off source period (120), provides sufficient independence of samples from a practical viewpoint (this is consistent with the results presented in [26]).

The state duration is a critical factor for the quality of the measurement process, therefore the information concerning measured mean, variance and their errors are gathered as a function of the number of samples, $N$. The analyzed variables related to the mean cell rate measurement are defined as follows:

1) measured average error of the measured mean instant rate:

$$
\bar{m}_{e}^{m}\left(N_{k}\right)=\frac{\sum \bar{\delta}_{k}^{m}\left(N_{k}\right)}{L_{N}}
$$

where $L_{N}$ expresses the size of the state population with $N_{k}$ samples;

2) measured variance of the error of the measured mean instant rate:

$$
\bar{V}_{e}^{m}\left(N_{k}\right)=\frac{\sum\left(\bar{\delta}_{k}^{m}\left(N_{k}\right)\right)^{2}}{L_{N}}-\left(\bar{m}_{e}^{m}\right)^{2}
$$

3) estimated variance of the error of the measured mean instant rate:

$$
\hat{V}_{e}^{m}\left(N_{k}\right)=\frac{\sum \bar{v}_{e, k}^{m}\left(N_{k}\right)}{L_{N}} .
$$

Fig. 4(a) depicts results obtained for the mean instant rate measurement. We observe that an underestimation appears for the short state periods. This spurious fault finds an explanation in the nature of the connection admission algorithm. When the state duration is short, the measured value is in general more likely to be significantly different from the declared one. In the figure, this fact is expressed by the error variance which indeed increases for small state durations. As a consequence, the overestimation of the mean cell rate results in an overestimation of the equivalent bandwidth, thus a new connection is more likely to be rejected (no state change), while in the opposite case (underestimation of the mean cell rate), a new connection is more likely to be accepted. This effect results in the fact that there are more short duration states with a corresponding underestimate of the mean cell rate. The correctness of the above reasoning is illustrated in Fig. 4(b). In this case, the admission decisions were made using the declared aggregated bandwidth instead of the estimated one. The bias caused by the feedback is significant only when the number of rejected connections is large as is the case in the chosen example. It can also be removed by an appropriate modification of the state duration definition (introduction of state changes when the connections are rejected). Note that in both instances the

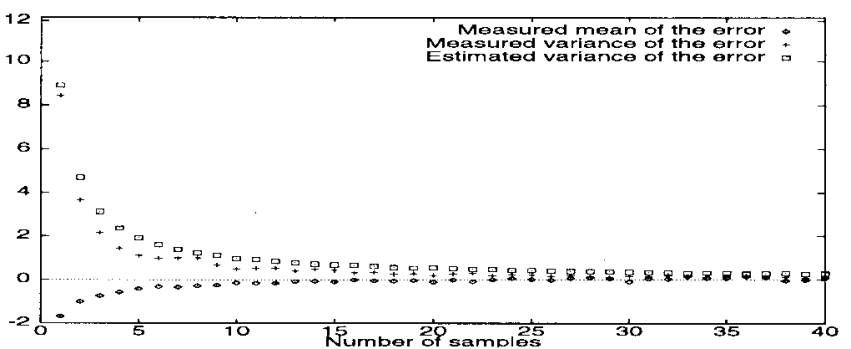

(a)

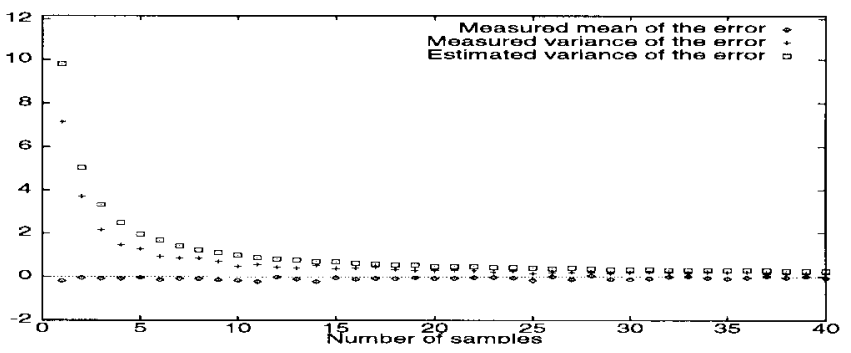

(b)

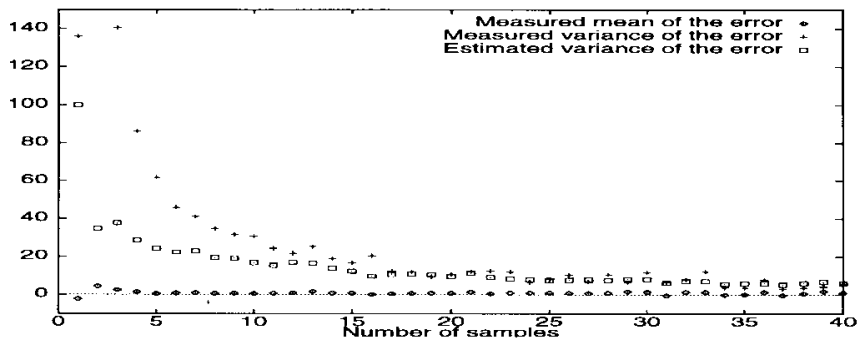

(c)

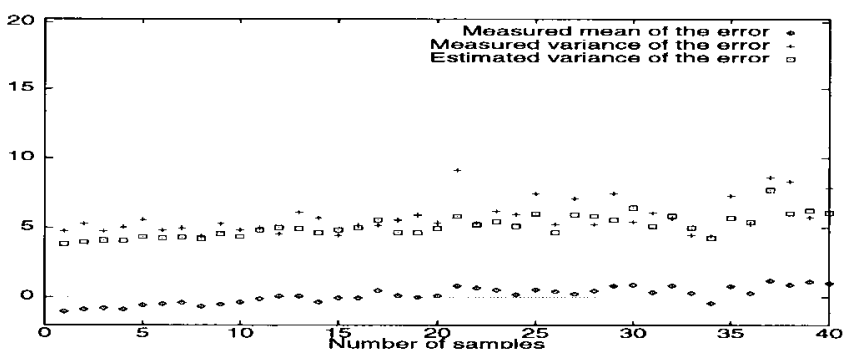

(d)

Fig. 4. (a), (b) Instant rate mean and (c), (d) variance measurement analysis.

variance of the error, estimated from (24), is close to the measured value.

Variables chosen to test the quality of cell rate variance measurement have been defined in an analogous way to those used for the cell rate mean measurement quality assessment:

1) measured average error of the measured instant rate variance:

$$
\bar{m}_{e}^{v}\left(N_{k}\right)=\frac{\sum \bar{\delta}_{k}^{v}\left(N_{k}\right)}{L_{N}} ;
$$

2) measured variance of the error of the measured instant rate variance:

$$
\bar{V}_{e}^{v}\left(N_{k}\right)=\frac{\sum\left(\bar{\delta}_{k}^{v}\left(N_{k}\right)\right)^{2}}{L_{N}}-\left(\bar{m}_{e}^{v}\right)^{2} ;
$$


3) estimated variance of the error of the measured instant rate variance:

$$
\hat{V}_{e}^{v}\left(N_{k}\right)=\frac{\sum \bar{v}_{e, k}^{v}\left(N_{k}\right)}{L_{N}}
$$

Results are presented in the Fig. 4(c). Discrepancy in the measured and estimated variance of the error for the small number of samples shows that, as could be expected, (18) cannot guarantee reliable results. To cope with the problem, we use an approximation based on a moving window with a fixed number of samples. Since the window span can contain several states, we applied the following approximation for the measured variance:

$$
\bar{V}_{k}=\frac{V_{k}^{d} \cdot \bar{V}_{k}^{w}}{V_{k}^{a}}
$$

where $V_{k}^{d}$ denotes the declared variance in the $k$ th state, $\bar{V}_{k}^{w}$ is the measured variance based on the samples from the whole window, and $V_{k}^{a}$ is the average declared variance in the window. The efficiency of the applied mechanism may be verified through the comparison of Fig. 4(c) (standard procedure) and Fig. 4(d) (procedure enhanced by the moving window with 40 samples), both simulations run under the same conditions. Improvement of the accuracy, particularly for the short state durations is significant.

\section{B. Mean and Variance Estimation Error}

Let us define the estimation errors for mean and variance of instant rate as

$$
\begin{aligned}
\hat{\delta}_{k}^{m} & =\hat{M}_{k}-M_{k} \\
\hat{\delta}_{k}^{v} & =\hat{V}_{k}-V_{k}
\end{aligned}
$$

respectively. Under the Gaussian assumption of the model and measurement errors, the estimation error distributions are also Gaussian with zero mean and variance defined by the diagonal elements of the error covariance matrix, $\hat{v}_{e, k}^{m}, \hat{v}_{e, k}^{v}$. The error covariance matrix is a function of the measurement and model errors [see, e.g., (8) and (9)]. The assessment of the measurement error was discussed in the previous section. In the following, we focus on the model error.

In general, the model error is a function of the declaration errors which are defined as a difference between the real and declared connection parameters

$$
c_{j}(t)=h_{j}^{r}(t)-h_{j}
$$

where time $t$ indicates that in general the error can be nonstationary. The transformation of the error into the discrete time domain can be done in several ways. One possibility is to assume $c_{k+i}=c\left(t_{k+i}\right) ; i=0,1, \cdots$, where $t_{k}$ is the time of connection acceptance. Another possibility is to use an average error over the discretization interval

$$
c_{j, k+i}=\frac{\int_{k+i}^{k+i+1} c_{j}(t)}{t_{k+i+1}-t_{k+i}} .
$$

In the context of our problem, the second option seems to be more appropriate since we are interested in QoS in the whole period between the state transitions. Then the model error is defined by

$$
e_{k}=c_{j, k}+\sum_{i}\left[c_{i, k}-c_{i, k-1}\right]
$$

where index $j$ corresponds to the new or departing connection and index $i$ corresponds to an existing connection. Observe that, in general, the periods between the state transitions are significantly shorter than the connection duration. This feature indicates that although the connection parameters might be different from declarations, one can expect that there will be very large autocorrelation between the values in the subsequent system states so the terms under summation in (37) will be small. Moreover, under the assumption of statistical independence of the connection instant rate processes, these terms will be positive and negative. These premises lead to the conclusion that the second term in (37) can be neglected so the model error can be approximated by the declaration error of the new or departing connection

$$
e_{k}=c_{j, k} \text {. }
$$

This approximation is exact when each connection process is stationary. Based on (38), the covariance matrix of the model error, $Q_{k}$, is defined by the predicted variances of declaration errors, $v_{e, k}^{m}, v_{e, k}^{v}$, for cell rate mean and variance, respectively. These values can be evaluated from statistics. In our model it is assumed that the declaration errors have Gaussian distribution with zero mean. Nevertheless, the algorithm can cope very well with distributions quite different from the Gaussian, as is shown in Section V.

To verify the accuracy of the instant rate mean and variance estimation we use the example from the previous section, with introduced declaration error. In this case, the source declared parameters, $m^{d}, v^{d}$, are still the same $\left(m^{d}=0.5, v^{d}=0.25\right)$, but the actual ones are generated with the Gaussian error with zero mean and variances: $v_{e, k}^{m}=0.05$ (for practical reasons the distribution is limited to the range $m \in[0.2,0.8]), v_{e, k}^{v}=$ 0.01 (in this case, the distribution is limited to the range $v \in[0.1,0.4])$. Note that due to the relation between the mean and variance, $v_{k}=m_{k} \cdot\left(P R_{k}-m_{k}\right)$, the peak rates, $P R_{k}$, have different values for each connection.

A set of the following variables will be used in the sequel:

1) the measured average error of the measured instant rate mean and variance, respectively,

$$
\bar{m}_{e}^{m}=E\left[\bar{M}_{k}-M_{k}\right] ; \quad \bar{m}_{e}^{v}=E\left[\bar{V}_{k}-V_{k}\right] ;
$$

2) the measured variance of the error of the measured instant rate mean and variance, respectively,

$$
\begin{aligned}
\bar{V}_{e}^{m} & =E\left[\left(\bar{M}_{k}-M_{k}\right)^{2}\right]-\left(\bar{m}_{e}^{m}\right)^{2} \\
\bar{V}_{e}^{v} & =E\left[\left(\bar{V}_{k}-V_{k}\right)^{2}\right]-\left(\bar{m}_{e}^{v}\right)^{2} ;
\end{aligned}
$$

3) the estimated variance of the error of the measured instant rate mean and variance, respectively,

$$
\hat{V}_{e}^{m}=E\left[\bar{v}_{e, k}^{m}\right] ; \quad \hat{V}_{e}^{v}=E\left[\bar{v}_{e, k}^{v}\right] ;
$$

4) the measured average error of the estimated instant rate mean and variance, respectively,

$$
\hat{m}_{e}^{m}=E\left[\hat{M}_{k}-M_{k}\right] ; \quad \hat{m}_{e}^{v}=E\left[\hat{V}_{k}-V_{k}\right] .
$$


TABLE I

Instant Rate Mean Estimation Results

\begin{tabular}{cccccc}
\hline Model & $\bar{m}_{e}^{m}$ & $\bar{M}$ & $\bar{V}_{e}^{m}$ & $\hat{V}_{e}^{m}$ & $\hat{m}_{e}^{m}$ \\
\hline 1 & -0.090 & 18.5 & 4.99 & 4.78 & -0.068 \\
2 & -0.091 & 18.5 & 4.69 & 4.79 & -0.064 \\
\hline
\end{tabular}

TABLE II

INSTANT RATE VARIANCE ESTIMATION RESUlTS

\begin{tabular}{cccccc}
\hline Model & $\bar{m}_{e}^{v}$ & $\bar{V}$ & $\bar{V}_{e}^{v}$ & $\hat{V}_{e}^{v}$ & $\hat{m}_{e}^{v}$ \\
\hline 1 & -0.053 & 9.27 & 4.39 & 4.09 & -0.733 \\
2 & -0.134 & 9.11 & 4.23 & 4.20 & -0.018 \\
\hline
\end{tabular}

TABLE III

ACCURACY OF EQUiVALENT BANDWIDTh Estimation

\begin{tabular}{ccccc}
\hline Model & $\hat{m}_{e}^{m}$ & $\hat{m}_{e}^{v}$ & $\bar{E}[\hat{G}-G]$ & $\bar{E}[\hat{G}]$ \\
\hline 2 & -0.020 & -0.003 & -0.243 & 22.1 \\
3 & -0.054 & -0.019 & -0.010 & 22.3 \\
\hline
\end{tabular}

The results corresponding to the instant rate mean and variance estimation are presented in Tables I and II, respectively. Model 1 corresponds to the algorithms and formulae presented in the previous section. The mean instant rate is estimated with a satisfactory accuracy, whereby the error does not exceed $0.5 \%$ of the estimated value. However, the results of the instant rate variance estimation are less satisfactory. We observe that the measurement part works appropriately, but the estimation produced a significant error. This can be explained by a strong correlation between the measured values of instant rate variance, $\bar{V}_{k}$, and variance of the instant rate variance error, $\bar{v}_{e, k}^{v}$ since the same set of samples is used to evaluate both values. As a result, whenever, for statistical reasons, the value of the measured variance is underestimated it has a larger weight in the Kalman filter due to the underestimated measurement error. This effect gives, on average, underestimation of the estimated variance of instant rate. This bias can be reduced by extending the window span used to evaluate the forth moment. The results for the extended window with $N_{\text {ext.win. }}=100$ samples are presented in Tables I and II (Model 2). The quality of variance measurement is noticeably improved.

\section{Equivalent Bandwidth Estimation Error}

The evaluation of the equivalent bandwidth estimation accuracy is based on the example from the previous section. The results concerning the error in equivalent bandwidth estimation are presented in the Table III (Model 2), where $\bar{E}[\cdot]$ denotes the measured average value. Note that although the mean and variance of the instant rate are estimated correctly, the equivalent bandwidth is slightly underestimated. This is due to the fact that the exact relation between the equivalent bandwidth and the instant rate mean and variance is slightly nonlinear and nonsymmetrical with respect to the central point of source parameter distribution while the applied approximation (14) is linear. To illustrate this effect, we evaluated "optimal" coefficients, $\theta, \gamma$, by integrating the exact equivalent bandwidth function. The results are given in the Table III (Model 3). In this case, the error is negligible.
TABLE IV

Quality of the Reserved EQuivalent Bandwidth Estimation

\begin{tabular}{cc}
\hline Imp. $\epsilon_{1}$ & $\hat{P}\left\{G_{k}>\hat{G}_{k}+R_{k}\right\}$ \\
\hline $10^{-1}$ & $3.2 \cdot 10^{-2}$ \\
$10^{-2}$ & $3.5 \cdot 10^{-3}$ \\
$10^{-3}$ & $4.7 \cdot 10^{-4}$ \\
$10^{-4}$ & $1.1 \cdot 10^{-4}$ \\
$10^{-5}$ & $1.3 \cdot 10^{-5}$ \\
\hline
\end{tabular}

To verify the accuracy of the bandwidth reserved for the estimation error, $R_{k}$, the probability $P\left\{G_{k}>\hat{G}_{k}+R_{k}\right\}$ was estimated in a simulation experiment. In order to reduce the declaration error distribution deformations caused by the physical constraints, the declaration error for variance was reduced: $v_{e, k}^{v}=0.001$. The estimated probabilities $\hat{P}\left\{G_{k}>\hat{G}_{k}+R_{k}\right\}$ are in Table IV for different values of the constraint $\epsilon_{1}$. Note that in all cases the estimated probabilities are close to the constraint defined by (2).

\section{CONNECTION ADMission AnALYsis}

\section{A. Connection Admission Procedure}

As stated in Section II, in the proposed framework a new connection is accepted if

$$
g_{k}^{\prime} \leq L-G_{k-1}^{\prime}
$$

Note that in this condition the estimate from state $k-1$ is treated as a prediction for state $k$. The implication is that the aggregate connection process can be treated as stationary over the period of the two states. While estimation of $G_{k-1}^{\prime}=$ $\hat{G}_{k-1}+R_{k-1}$ was already described, interpretation and evaluation of the bandwidth reserved for a new connection, $g_{k}^{\prime}$, requires additional clarification. In particular, it is important to define more precisely what is the design objective of the connection admission procedure and how the quality of this procedure should be judged. The answers to these questions are not straightforward. Note that the main criterion of the connection admission is to ensure that the QoS constraint is met (on the cell layer). In our model, this requirement is fulfilled when the real bandwidth required by the admitted connections does not exceed the link capacity, $G_{k} \leq L$. Obviously strict execution of this condition might cause the bandwidth utilization to be compromised. Thus, to improve bandwidth utilization, we allow that $G_{k}>L$ with a certain small probability

$$
P\left\{G_{k}>L\right\} \leq \epsilon_{2} .
$$

There is one drawback with this formulation. Namely, this probability depends strongly on the connection arrival process and connection bandwidth requirements. In particular, the smaller traffic level the smaller $P\left\{G_{k}>L\right\}$. This feature indicates that the condition (44) is not convenient for the connection admission algorithm design.

To deal with this issue, we propose another definition of the connection admission procedure quality which is independent from the connection arrival process and connection bandwidth requirements. It is based on the following conditional probability:

$$
P\left\{G_{k}>L \mid g_{k}^{\prime}=L-G_{k-1}^{\prime}\right\} \leq \epsilon_{2} .
$$


In this case, the quality is defined for the critical case where the residual capacity is equal to the one required by a new connection. Observe that condition (45) is also fulfilled when

$$
P\left\{G_{k}>g_{k}^{\prime}+G_{k-1}^{\prime}\right\} \leq \epsilon_{2} .
$$

The latter condition constitutes the basis for design and evaluation of the proposed connection admission procedure.

In analogy with $G_{k-1}^{\prime}$, the bandwidth reserved for a new connection, $g_{k}^{\prime}$, can be decomposed into two parts, the equivalent bandwidth, $g_{k}^{d}$, evaluated from the declared parameters and the bandwidth, $r_{k}$, reserved for the declaration error. Note that the sum $r_{k}+R_{k-1}$ could be evaluated from the superposition of distributions of $g_{k}$ and $G_{k-1}$. Nevertheless, from the connection admission viewpoint it is more convenient to separate evaluation of $r_{k}$ from evaluation of $R_{k-1}$. The main reason behind this approach is that in this case the processing of the CAC control cell in a transit ATM node is limited to a simple comparison of two numbers (otherwise, some more complex calculation would have to be performed).

Based on the Gaussian assumption, the bandwidth reserved for the declaration error can be evaluated from

$$
r_{k}=U\left(\epsilon_{3}\right) \sqrt{\gamma^{2} v_{e}^{m}+\theta^{2} v_{e}^{v}}
$$

where the value of the constraint $\epsilon_{3}$ can be chosen between $\epsilon_{3}=\epsilon_{2}-\epsilon_{1}$ (conservative) and $\epsilon_{3}=\epsilon_{2} / \epsilon_{1}$ (optimistic). Another possibility is to apply an approach where the parameter $r_{k}$ is defined by the connection peak rate, $P R_{k}$,

$$
r_{k}=P R_{k}-g_{k}^{d}
$$

This approach is simpler and safer since the peak rate defines the upper boundary for the error. In the following, we use the second approach.

\section{B. Numerical Examples}

We start by describing a simulation model used for assessment of the proposed algorithms. To avoid excessive complexity we simplified the simulation model as much as possible to concentrate on the main issues. In particular, only the instant rate layer is modeled and the link buffer has zero length. It should be stressed that the buffer-less case was chosen only to simplify evaluation of the exact equivalent bandwidth allocation. This choice does not restrict the analyzed CAC model applications nor limit generality of the results. This follows from the fact that the CAC algorithm operates on the notion of the equivalent bandwidth in a way which separates the issue of CAC adaptiveness from buffer dimensioning and performance measure on the cell layer. Moreover, the buffer-less case exemplifies the burst scale layer model whose performance is critical for a robust CAC algorithms for real-time services.

The requests for connections of particular class are generated with intensity $\lambda$ (Poissonian distribution) and mean holding time $\mu^{-1}$ (exponential distribution). The connections are of on-off type and are described by the peak rate, $P R$, average burst length, $B$, (with programmable distribution) and the average silence length, $S$ (exponential distribution). The QoS constraint (cell loss probability $-\mathcal{B}^{c}$ ) is set to a relatively high value $\mathcal{B}^{c}=10^{-2}$ in order to achieve a reliable estimate of the cell loss probability distribution under nonstationary traffic conditions. The estimation error constraint is set to the same value, $\epsilon_{1}=10^{-2}$.

The declaration error can be generated in many ways. The results presented in the previous section (henceforth, referred to as Ex.1) are based on the Gaussian model for this error distribution, which conforms with the Kalman filter assumptions. In this section, we introduce another error model which is significantly different from the Kalman filter assumption. Namely, the error generator has two cyclic states ("on" and "off") with the same period $T$. When the generator is in the state "on" all connections accepted in this state are generated with the burst length larger than the declared one $\left(B^{\prime}=B+\Delta B\right)$. In the "off" state, all new connections are generated with the declared burst length (for entire duration of the connection). Note that in this case the error has no zero mean. The reason for this model is to evaluate the adaptation scheme under more stressing conditions.

The binomial distribution is used to evaluate both the equivalent bandwidth from the source declarations, $g_{k}^{d}=$ $f_{g}\left(h_{k}\right)$, and the exact aggregate equivalent bandwidth based on the real connection parameters parameters, $G_{k}$ (to assess the accuracy of the adaptation scheme).

The performance of the CAC mechanism under the deterministic and nonstationary error is illustrated on two examples. The first one (Ex.2) is defined by: link and connection parameters- $L=25, \lambda=200^{-1}, \mu^{-1}=5 \cdot 10^{4}$; source parameters- $P R=1, B=50, S=70, v_{e}^{m}=0.05, v_{e}^{v}=$ 0.002 ; error generator parameters $-T=2 \cdot 10^{5}, B^{\prime}=100$.

A sample of the equivalent bandwidth allocation dynamics, during a part of the simulation run, is presented in Fig. 5. In Fig. 5(a), the total number of connections and the number of connections with modified burst length are given. During the "on" period of the error generator the parameters of almost all connections are modified while at the end of the "off" period almost all connections have the declared parameters.

The trajectory of the real aggregate equivalent bandwidth, $G_{k}$, and the difference between the estimated and real aggregate equivalent bandwidth, $\hat{G}_{k}-G_{k}$, are depicted in Fig. 5(b). The estimated aggregate equivalent bandwidth accurately tracks the actual value. It can be noticed that the largest underestimation of the equivalent bandwidth occurs during the "on" period of the error generator. Nevertheless the bandwidth reserved for estimation error ensures that the real aggregate equivalent bandwidth does not exceed the link capacity. This result shows that the proposed scheme is very robust since during the error generation period the real declaration error is not only on average two times larger than that declared but also is always positive. This robustness results from two factors. First, at the moment of a new connection admission there is provision for the equivalent bandwidth equal to its peak rate so no matter how malicious the source, the QoS is kept under the constraint during the new state. Although in subsequent states this provision disappears (the connection is included in the aggregate equivalent bandwidth), the system has time to correct the bandwidth allocation by means of the measurement process incorporated in the estimation of the aggregate equivalent bandwidth. 


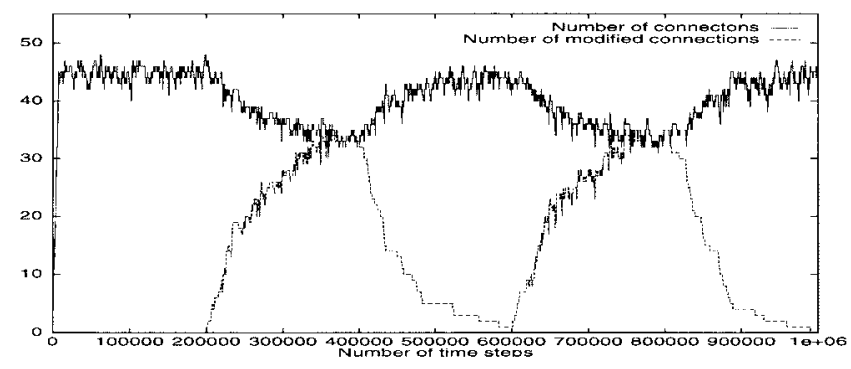

(a)



(b)

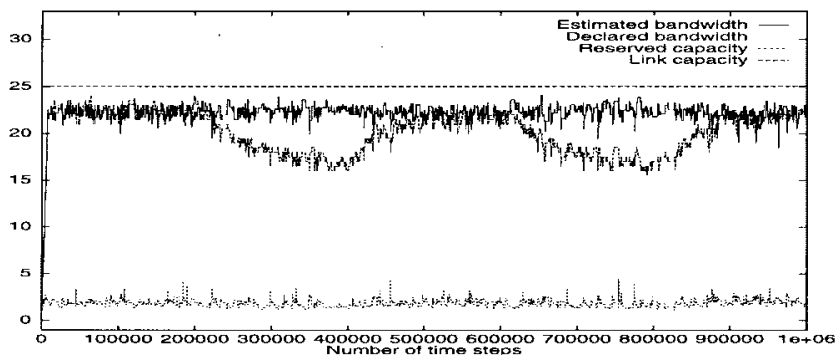

(c)

Fig. 5. Trajectories of the system variables versus simulation time (Ex.2).

The efficiency of the proposed adaptive algorithm is demonstrated in Fig. 5(c), where the estimated aggregate equivalent bandwidth allocation, $\hat{G}_{k}$, and the declared aggregate equivalent bandwidth, $G_{k}^{d}$ are plotted. The large gap between the two trajectories, in the periods when the number of modified connections is significant, indicates that the connection admission scheme based solely on the declared parameter would allow too many connections and the real aggregate equivalent bandwidth would significantly exceed the allocated capacity. In addition, the bandwidth reserved for the estimation error $R_{k}$ is depicted in Fig. 5(c).

The second example (Ex.3) with deterministic and nonstationary error includes two connection classes and is defined by: link and connection parameters- $L=50, \lambda_{1}=$ $1000^{-1}, \mu_{1}^{-1}=5 \cdot 10^{4}, \lambda_{2}=500^{-1}, \mu_{2}^{-1}=6 \cdot 10^{4}$; source parameters- $P R_{1}=1, B_{1}=50, S_{1}=70, v_{e, 1}^{m}=0.1, v_{e, 1}^{v}=$ $0.1, P R_{2}=4, B_{2}=20, S_{2}=80, v_{e, 2}^{m}=0.2, v_{e, 2}^{v}=0.2$; error generator parameters- $T=10^{5}, B_{1}^{\prime}=150, B_{2}^{\prime}=60$.

A sample of the system dynamics, during a part of the simulation run, is presented in Fig. 6. In Fig. 6(a), the trajectories of connection numbers are given. The trajectories of the estimated aggregate equivalent bandwidth, $\hat{G}_{k}$, and the declared aggregate equivalent bandwidth, $G_{k}^{d}$, are presented in Fig. 6(b). The trajectory of the cell loss probability (estimated in the time windows used for the cell variance measurements)

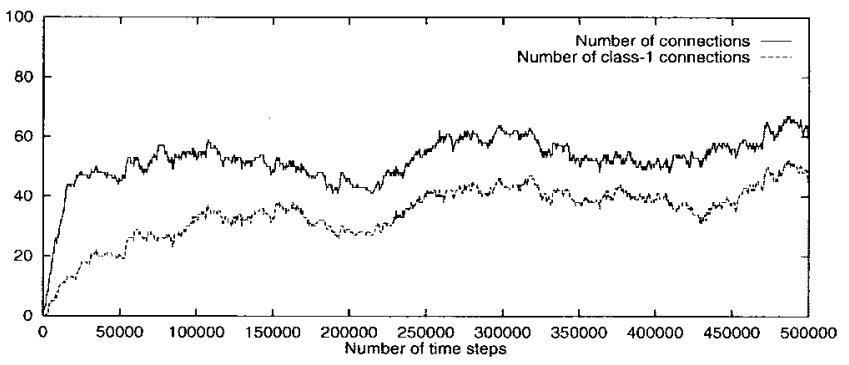

(a)



(b)



(c)

Fig. 6. Trajectories of the system variables versus simulation time (Ex.3).

is given in Fig. 6(c). The results show that the algorithm is also robust in the multiclass environment.

\section{System with Policing Mechanism}

In this section, we consider the case where the source parameters are controlled by means of the policing mechanism. Observe that, in general, introduction of the policing mechanism reduces the magnitude of the source declaration error seen at the switch output ports. Thus, there is a potential to increase further the link bandwidth utilization without compromising the QoS performance. In the following we will extend the adaptive algorithm presented in the previous sections to take advantage of the policing mechanism. We will also give numerical examples to illustrate the accuracy of this approach and to discuss the advantages and disadvantages of the source parameter enforcement.

We start from the assumption that the source declaration errors are Gaussian with zero mean. When there is no policing mechanism, this assumption provides optimal conditions for the aggregate bandwidth estimation based on Kalman filter. The introduction of the policing mechanism implies that, in general, the connection process entering the link can be different from the source process. In particular, it means that the declaration error distributions are no longer Gaussian and, 


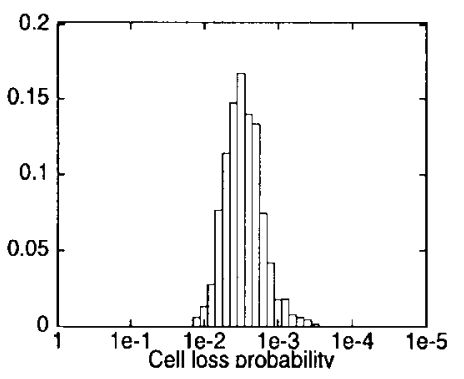

(a)

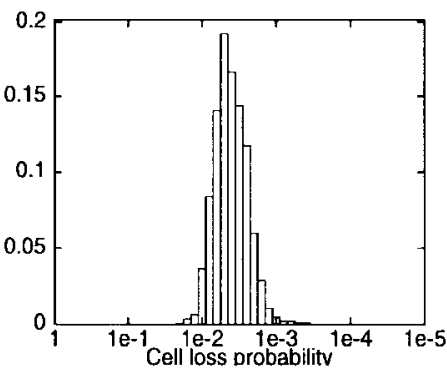

(b)



(c)

Fig. 7. Cell loss probability distributions, Ex.1: (a) $L R=1.0$, (b) $L R=0.62$, and (c) $L R=0.50$.



(a)

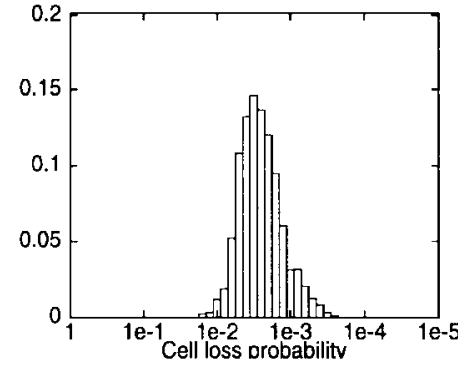

(b)

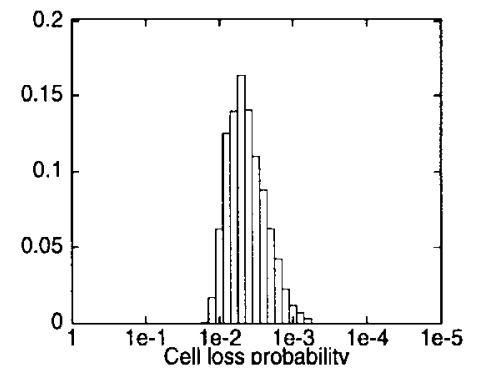

(c)

Fig. 8. Cell loss probability distributions, Ex.2: (a) $L R=1.0$, (b) $L R=0.62$, and (c) $L R=0.50$ ).

in addition, they are not symmetrical (nonzero mean). To cope with this issue, in the following, we describe an approximation which enables the basic connection admission algorithm to be preserved with only a few minor modifications. This is achieved by a modification of the declared parameters concerning the mean cell rate with the result that the declaration error has zero mean. Then these parameters are used in the original algorithm which assumes Gaussian error distributions.

The influence of the policing mechanism on the mean rate declaration error distribution can be modeled as follows. First note that the upper Gaussian distribution tail of the original distribution is truncated at the threshold value which is defined by the leak rate, $L R$, of the policing mechanism. Then we assume that all connections with source mean cell rate higher than the threshold have mean cell rate equal to the threshold after the policing mechanism. Concerning the connections with the source mean cell rate smaller than the threshold we assume that their mean cell rate is preserved. While the two assumptions are approximations, they have the advantage of simplifying the model and being conservative. Based on these assumptions and the source declared parameters we can numerically evaluate the mean $m_{e, k}^{m^{\prime}}$ and variance $v_{e, k}^{m^{\prime}}$ of the modified error distribution. Then the modified declared connection mean cell rate, used by the original connection admission procedure, is defined as

$$
m_{k}^{\prime}=m_{k}+m_{e, k}^{m^{\prime}}
$$

and the mean rate declaration error is assumed to have Gaussian distribution with zero mean and variance $v_{e, k}^{m^{\prime}}$.

Note, that one could try to apply an analogous procedure to modify the declared parameters concerning the variance of the cell rate. However, since the sensitivity of the estimation procedure with respect to the change in rate variance parameters caused by policing mechanism is less significant compared to the change in mean rate parameters, we use the source's declared rate variance and its error variance in the studied CAC procedure.

The modifications of the declared mean rate and the declaration error variance influence the evaluation of the aggregate equivalent bandwidth and the bandwidth reserved for the estimation error. The policing mechanism also influences the bandwidth reserved for the declaration error, $r_{k}$. In the original approach, this bandwidth was evaluated as the difference between the connection peak rate and equivalent bandwidth (48). Since the policing mechanism limits the worst-case equivalent bandwidth used by the connection, the bandwidth reserved for the declaration error can be reduced to

$$
r_{k}^{\prime}=g_{k}^{\max }-g_{k}^{d}
$$

where $g_{k}^{\max }$ denotes the equivalent bandwidth required by the worst-case source.

\section{A. Numerical Examples}

Accuracy and performance of the described approximations for different policing thresholds are illustrated in Table $\mathrm{V}$ and Fig. 7 for Ex.1 (Gaussian distribution of the declaration error) and in Table VI and Fig. 8 for Ex.2 (deterministic and nonstationary declaration error). Three different thresholds for mean cell rate are applied to each example: 1.0 (peak rate limitation only), 0.51 and 0.41 (the declared mean cell rate). The design of the policing mechanism was based on the model presented in [28] (cell rejection rate for conforming source $\left.-10^{-3}\right)$. The corresponding leak rates are 1.0, 0.62, and 0.5 respectively. The cell loss probabilities are estimated in the windows used for the cell variance measurements. The average and the distribution of cell loss probabilities are given in Tables V and VI, and Figs 7 and 8, respectively. The performance of the CAC is very close to the constraints $\left(\mathcal{B}^{c}=0.01, \epsilon_{1}=0.01\right)$ in all cases. This result shows that 
TABLE V

Average Values (Ex. 1)

\begin{tabular}{lccc}
\hline Policed mean cell rate & 1.0 & 0.51 & 0.41 \\
Leak rate & 1.0 & 0.62 & 0.50 \\
\hline reserved bandwidth & 1.54 & 1.34 & 1.25 \\
ag. equivalent band. & 22.2 & 22.8 & 22.9 \\
cell loss probability & $3.5 \mathrm{e}-03$ & $4.7 \mathrm{e}-03$ & $5.7 \mathrm{e}-03$ \\
number of connections & 46.2 & 49.0 & 52.4 \\
\hline
\end{tabular}

TABLE VI

Average Values (Ex.2)

\begin{tabular}{lccc}
\hline Policed mean cell rate & 1.0 & 0.51 & 0.41 \\
Leak rate & 1.0 & 0.62 & 0.50 \\
\hline reserved bandwidth & 1.39 & 1.25 & 0.56 \\
ag. equivalent band. & 22.3 & 22.8 & 23.5 \\
cell loss probability & $1.8 \mathrm{e}-03$ & $3.2 \mathrm{e}-03$ & $5.0 \mathrm{e}-03$ \\
number of connections & 39.2 & 41.6 & 44.6 \\
\hline
\end{tabular}

the described approximation is accurate and that the adaptive CAC can work efficiently with the policing mechanism.

As could be predicted, the more strict policing the more connections can be accepted on average. Nevertheless, this feature does not necessarily constitute an advantage. First, the increase in the number of accepted connections is achieved at the expense of the source parameter limitation which can be seen as an unwanted restriction from the user viewpoint. This restriction can force users to declare increased parameters, since in many cases the source does not accurately know their parameters in advance. Thus this behavior can reduce bandwidth utilization. Also we observe that the average aggregate bandwidth is similar in all cases, especially in the Ex.1. Thus if the tariff is proportional to the actual bandwidth usage, the network revenue is not affected significantly. Note that introduction of a tariff, which charges more for the traffic exceeding the declaration, can motivate users to declare parameters as accurately as possible (see [27]).

\section{CONCLUSION}

Most of the existing traffic control and bandwidth management algorithms for ATM based networks focus on particular types of services. In the paper, we have proposed a unified framework which bridges the gap between the algorithms for real-time services and controllable data services. The framework is based on the rate-based scheme for controllable data services, recommended by the ATM-Forum, and the adaptive connection admission algorithm proposed in the paper. Both algorithms react to measurements of the cell rate process at the switch output ports and use the same data base structure and signaling protocol. Besides reduction of the algorithms complexity and cost this feature can also increase bandwidth utilization by close coordination of both algorithms.

The central concept of the proposed connection admission algorithm is estimation of the aggregate equivalent bandwidth required by connections carried in each output port of the ATM switches. The estimation process takes into account both the traffic source declarations and the connection superposition process measurements in the switch output ports. This is done in an optimization framework using a linear Kalman filter. The
Kalman filter approach fits naturally the estimation problem under consideration. It takes into account the connection level dynamics of the system in an optimal way and it gives information for evaluation of bandwidth reserved for possible estimation error. This bandwidth ensures a statistical guarantee for quality of service.

The numerical analysis of the estimation process showed that the bandwidth reserved for the estimation error is very accurate when the declaration error has Gaussian distribution. The study of the connection admission algorithm under nonstationary conditions and large, non-Gaussian, declaration errors demonstrated that the approach is very robust and copes very well with undeclared changes in traffic parameters.

By applying an efficient approximation, the estimation model was extended for cases with source policing algorithms. The numerical study illustrated the tradeoff between relaxed and strict source policing. The results indicate that application of relaxed policing can be advantageous from both user and network viewpoints. The inclusion of a policing mechanism also shows that the proposed approach can coexist with the ATM Forum and ITU recommendations.

There are several further model extensions which could be investigated. For example, the measurement process constitutes an important area for study where declared and measured autocorrelation functions can play an important role. More complex filters can be tried to check whether it is possible to estimate some characteristics corresponding more directly to the QoS metrics. Also the problem of correlated sources can be investigated since the model can handle such cases. Finally an integrated model for bandwidth management for CTP and NCTP services based on a partly common estimation algorithm can be studied.

\section{ACKNOWLEDGMENT}

The authors wish to thank M. Jia for his contribution concerning the measurement model. The authors are also grateful to B. V. Shukhman and O. Montanuy for their help in the numerical study. Finally, the authors would like to acknowledge the effort of anonymous reviewers whose remarks have influenced the final shape of this paper.

\section{REFERENCES}

[1] J. S. Turner, "The challenge of multi-point communication," in 5th ITC Seminar, Lake Como, Italy, May 1987.

[2] G. Woodruff, R. Kositpaiboon, G. Fitzpatrick, and P. Richards, "Control of ATM statistical multiplexing performance," in Proc. ITC Specialist Seminar, Adelaide, Australia, 1989.

[3] Z. Dziong, J. Choquette, K.-Q. Liao, and L. Mason, "Admission control and routing in ATM networks," in Proc. ITC Specialist Seminar, Adelaide, Sept. 1989.

[4] R. Guerin, H. Ahmadi, and M. Naghshineh, "Equivalent capacity and its application to bandwidth allocation in high-speed networks," IEEE J. Select. Areas Commun., vol. 9, 1991.

[5] F. Kelly, "Effective bandwidths at multi-class queues," Queueing Syst., vol. 9, pp. 5-15, 1991.

[6] R. Jain, "Congestion control and traffic management in ATM networks: recent advances and a survey," Computer Networks ISDN Syst., Jan. 1995.

[7] D. Mitra, "Asymptotically optimal design of congestion control for high speed data networks," IEEE Trans. Commun., vol. 40, pp. 301-311, Feb. 1992. 
[8] K.-Q. Liao and L. G. Mason, "A congestion control framework for broadband ISDN using selective window Control," in Proc. Broadband Communications'94, Paris, France, Mar. 1994.

[9] T. E. Tedijanto and L. Gun, "Effectiveness of dynamic bandwidth management mechanisms in ATM networks," Proc. INFOCOM'93, San Francisco, CA, 1993.

[10] H Saito., "Dynamic call admission control in ATM networks," IEEE J. Select. Areas Commun., vol. 9, Dec. 1991.

[11] H. Kroner and T. Renger, and R. Knobling, "Performance modeling of an adaptive CAC strategy for ATM networks," in Proc. ITC-14, Paris, France, June 1994.

[12] R. Griffiths and P. Key, "Adaptive call admission control in ATM networks," in Proc. ITC-14, Paris, France, June 1994.

[13] R. J. Gibbens, F. P. Kelly, and P. B. Key, "A decision-theoretic approach to call admission control in ATM networks," IEEE J. Select. Areas Commun., vol. 13, pp. 1101-1114, Aug. 1995.

[14] S.-Q. Li, S. Chong, and C. L. Hwang, "Link capacity allocation and network control by filtered input rate in high-speed networks," IEEE/ACM Trans. Networking, vol. 3, Feb. 1995.

[15] C. D. Pack and B. A. Whitaker, "Kalman filter models for network forecasting," Bell Syst. Tech. J., vol. 61, no. 1, pp. 1-14, 1982.

[16] P. Chemouil and J. Filipiak, "Kalman filtering of traffic fluctuations for real-time network management," Annales des Telecommun., vol. 44, nos. 11/12, pp. 633-640, 1989.

[17] Z. Dziong and L. Mason, "Call admission and routing in multi-service loss networks," IEEE Trans. Commun., vol. 42, Apr. 1994.

[18] P. E. Boyer and D. P. Tranchier, "A reservation principle with applications to the ATM traffic control," Computer Networks ISDN Syst., vol. 24, pp. 321-334, 1992.

[19] J.W. Roberts, "Performance evaluation and design of multi-service networks," COST 224 final report Oct. 1992.

[20] G. Gallassi, G. Rigolio, and L. Fratta, "Bandwidth assignment and bandwidth enforcement policies," in Proc. GLOBECOM'89, Dallas, TX, 1989.

[21] Z. Dziong, K-Q. Liao, and L. Mason, "Effective bandwidth allocation and buffer dimensioning in atm based networks with priorities," Computer Networks ISDN-Systems, vol. 25, pp. 1065-1078, May 1993.

[22] M. Veeraraghavan, T. F. La Porta, and W. S. Lai, "An alternative approach to call/connection control in broadband switching systems," in IEEE BSS'95, Poznan, Poland, Apr. 1995.

[23] A. Gelb, Applied Optimal Estimation. Cambridge, MA: MIT Press, 1974.

[24] A. Gach, C. Mialaret, and P.E. Allard, "An Experimental Evaluation of Call Acceptance Management Algorithms in ATM Based Networks," in Proc. CCECE-92, Toronto, Ont., Canada, Sept. 1992.

[25] M. Ritter and P. Tran-Gia, Eds., COST 242 Mid Term Seminar, l'Aquila, 27-28 Sept. 1994.

[26] H. Yamada and S. Sumita, "A Traffic Measurement Method and its Application for Cell Loss Probability Estimation in ATM Networks," IEEE J. Select. Areas Commun., vol. 9, pp. 315-324, Dec. 1991.

[27] F. Kelly, "Tariffs and effective bandwidths in multiservice networks," in Proc. ITC-14, Antibes Juan-les-Pins, France, June 1994.

[28] K-Q Liao., Z. Dziong, L. Mason, and N. Tetreault, "Effectiveness of leaky bucket policing mechanism," in Proc. ICC'92, Chicago, IL, 1992.

[29] Z. Dziong, M. Juda, A. Jajszczyk, and L. G. Mason, "Influence of UPC on connection admission based on measurements in ATM switch output ports," in 1st Workshop ATM Traffic Management WATM'95, Paris, France, 1995, pp. 77-84.

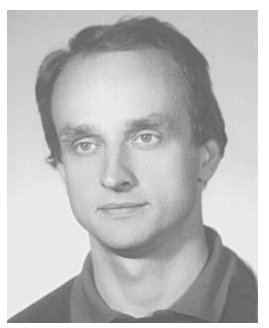

Zbigniew Dziong received the M.Sc. and Ph.D degrees from the Warsaw University of Technology, Poland, in 1974 and 1980, respectively, both in electrical engineering.

From 1974 to 1991, he was with the Warsaw University of Technology, Poland, as an Assistant Professor. He was on sabbatical leaves at the Centre National d'Etudes des Telecommunications, Paris, France, and at the Department of Communication Systems, Lund Institute of Technology, Sweden. Currently, he is with INRS-Telecommunications, Canada, as a Professor. He also helds a position of Research Fellow at the Canadian Institute of Telecommunication Research. His research interests include design, management and control of communication networks.

Dr. Dziong was co-recipient of the 1993 STENTOR Award for collaborative research in telecommunications for his contributions in the area of statedependent routing.

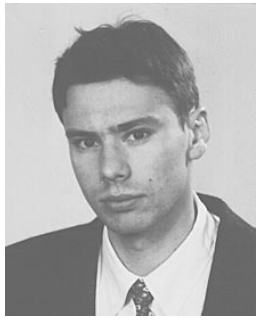

Marek Juda received the M.S. degree in electrical engineering and Diplome d'Ingenieur from The Franco-Polish School of New Information and Communication Technologies, Poznan, Poland, in 1995.

In 1994, he had an Intership with INRSTelecommunications, Montreal, Canada, where he was involved in research concerning adaptive bandwidth management in ATM networks. Since September 1995, he has been working with Andersen Consulting's Warsaw-based practice, where his primary interest is in network solutions and the communications industry.



Lorne G. Mason received the B.Sc. and Ph.D. degrees from the University of Saskatchewan, Canada, both in mechanical engineering, in 1963 and 1972, respectively.

He was with Bristol Aerojet, Winnipeg, Manitoba, from 1963 to 1965 , where he was involved in the design of the Black Brant rockets. He joined the British Columbia Telephone Company as a Traffic Engineer in 1966 and again in 1972 as a Consultant for planning digital networks. In 1973, he served as a Consultant to Yale University, where he, in collaboration with Prof. K. S. Narendra, pioneered the use of learning automata for adaptive routing in telecommunication networks. Between 1974 and 1977 , he was with Bell-Northern Research, Ottawa, Canada, where he developed planning tools and methods for digital network evolution and state-dependent routing. Since 1977, he has been with INRS-Télécommunications, where he currently holds the title of Full Professor. He also holds a position of "Professeur Associé" at ENST (Télécom Paris). His primary research interests are in the application of control theory and operations research methods to telecommunication network design and management. He has held numerous industrial research contracts and NSERC strategic grants in the area of broadband network design and analysis and has numerous publications on the subject. He is a participant in the Network of Centers of Excellence program sponsored by the Canadian government, where he is Project Leader on broadband network control. He has authored or co-authored over 60 papers on broadband comunications and is editor of the Proceedings of the IFIP-IEEE sponsored Conference "Broadband Communications '96," where he served as General Chairman.

Dr. Mason was co-recipient of the 1993 STENTOR Award for collaborative research in telecommunications for his contributions to state-dependent routing. 